\title{
Komplementarität: Ergänzungsfähigkeit und Gegensätzlichkeit
}

\author{
Bettina Reiter \\ Akademie für Ganzheitsmedizin, Wien, Österreich
}

\begin{abstract}
Die Medizin ist eine soziale Wissenschaft, und die Politik ist nichts weiter als Medizin im Großen. Rudolf von Virchow
\end{abstract}

Was ist eigentlich das Komplementäre an der Komplementärmedizin? Bezieht sich dieser Begriff nicht nur auf die Ergänzungsfähigkeit der Komplementärmedizin gegenüber dem (be)herrschenden Paradigma der akademischen Medizin, sozusagen in ihrer Potentialität zum Anschmiegen, Einfügen und Sich-mit-den-Lücken-Begnügen?

Komplementarität heißt in der Erkenntnistheorie die Zusammengehörigkeit eigentlich separater, ja sogar gegensätzlicher Begriffe wie etwa der Kräfte Yin und Yang im Taoismus oder, medizinischer, die Annahme von gänzlich seelischen und gänzlich körperlichen Faktoren bei der Entstehung von psychosomatischen Leiden. Das eine kann nie das andere werden, aber ohne das andere auch nicht sein: Am ultimativen Yang-Punkt beginnt Yin. Eine psychosomatische Krankheit braucht als Entstehungsbedingung sowohl die somatische als auch die psychische Leidensebene. So radikal wird das Komplementäre der Komplementärmedizin selten verstanden, jedenfalls nicht, wenn es um die Außendarstellung geht. Gibt es innerhalb des Paradigmas, und auch besonders in ForSCHENDE KomplementÄRMEdizIN als Flaggschiff einer offenen Diskussionskultur, dazu natürlich eine - zuweilen sogar hitzige geführte - Diskussion um erkenntnistheoretische Vorstellungen zur Wirkungsweise von komplexen medizinischen Interventionen, wie es ja alle komplementärmedizinischen Verfahren sind [1, 2], so nehmen die Angriffslust und das Selbstbewusstsein rapide ab, je näher die klassische akademische Medizin und ihre Publikationsorgane rücken und damit der Rechtfertigungsdruck auf die Komplementärmedizin, die sich dann gerne selbst als «ja nur ergänzend» sieht und quasi bittet, aus dem großen Haus der Gesamtmedizin nicht hinausgeworfen zu werden, auch wenn sie in den allermeisten Fällen «nur» Evidenzgrade 3 und/oder 4 für ihre Behandlungsstrategien vorweisen kann. Dass das Match von vorneherein unfair geführt wird, da die Komplementärmedizin bei Weitem nicht über die gleichen humanen, akademischen und finanziellen Ressourcen verfügt wie die akademische Medizin, wird zwar manchmal aufgezeigt [3], spielt aber eine erstaunlich kleine Rolle in der generellen Diskussion.

Das gilt genauso für den anderen Aspekt des Begriffs Komplementarität, der den notwendigen inneren Gegensatz zweier zusammengehöriger Einheiten betont, wie in den beiden Beispielen am Anfang, am prominentesten und folgenreichsten aber natürlich im quantenphysikalischen WelleTeilchen-Modell der Materie.

Gerd Gigerenzer, Leiter des Max-Planck-Instituts für Bildungsforschung, das mit den PISA-Studien ja sehr bekannt wurde, hat sich jahrzehntelang dafür interessiert, wie Menschen ihre Entscheidungen treffen. Darüber hat er 2007 ein populäres Buch geschrieben, das - wie ich finde, zu Recht zum «Wissenschaftsbuch des Jahres 2007» gewählt worden ist: «Bauchentscheidungen - die Intelligenz des Unbewussten und die Macht der Intuition» [4] ist ein erfrischendes und oft überraschendes Plädoyer für die nicht rationale, alle Risiken abwägende Entscheidung nach dem Franklin-Muster (Schreibe die Vor- und Nachteile in eine Liste und bewerte dann die einzelnen Punkte). Intuitive Entscheidungen sind, auch statistisch gesehen, meist besser, das heißt, näher an der Wirklichkeit und dem zu erreichenden Ziel. Zum Beispiel ließ sich in einer Untersuchung an einem amerikanischen Krankenhaus die Frage: «Welcher Patient mit unklaren Beschwerden kommt auf eine Intensivstation zur kardialen Überwachung und welcher nicht?», mit der Anwendung eines komplizierten

\section{KARGER}

Fax +497614520714

Information@Karger.de

www.karger.com (c) 2009 S. Karger GmbH, Freiburg

Accessible online at:

www.karger.com/fok
Dr. med Bettina Reiter

Akademie für Ganzheitsmedizin

Sanatoriumstr. 2

1140 Wien, Österreich

Tel. +43 1688 7507-0, Fax -15

reiter@gamed.or.at 
Diagnosetools inklusive Taschenrechner zum Errechnen des individuellen Risikos auf einen Herzinfarkt nicht treffsicherer beantworten als mit der «bloßen» Intuition der Behandler [4, p $180 \mathrm{ff}$. Und letztere ist auf jeden Fall einfacher zu haben.

Das sind gute Nachrichten für eine selbstbewusste Komplementärmedizin, die den Begriff der Komplementarität als «ergänzend in der Gegensätzlichkeit» und nicht als «Ergänzung eines Mangels» oder als «Erweiterung eines Spektrums» auffassen will. Intuition war ja immer schon eine Ingredienz ärztlichen Handelns, im berühmten «klinischen Blick» des erfahrenen (und manchmal auch nur eingebildeten) Arztes ein Teil des auch ideologischen Nimbus des Heilers und Weisen, oft genug karikiert (bei Mozart, Molière, Nestroy ...) und im Zeitalter der evidenzbasierten Medizin (EbM) überhaupt abgeschafft, da von Evidenz ersetzt. Kognitionspsychologische Arbeiten, wie die von Gigerenzer in seinem Buch zusammengetragenen und dargelegten, schlagen nun eine Rehabilitation der Intuition, des «Bauchgefühls», vor - und darüber hinaus die systematische Einarbeitung intuitiver Möglichkeiten in erkenntnis- und handlungsorientierten Gebiete, von denen hier logischerweise die Medizin am meisten interessiert. In der aktuellen Diskussion um Wirksamkeitsparameter und deren Bewertung in der EbMHierarchie und vor allem auch epistemischen Eingleisigkeit der EbM, der Diskussion um Kontextfaktoren und die Neubewertung und Einordnung der als «Placebo-Gruppe» zusammenfassbaren Phänomene, mag und wird die Intuition wieder ihren Platz finden - mit dem entscheidenden Unterschied, dass Forschungen wie die erwähnten kognitionspsychologischen den Begriff zur weiteren Diskussion durch ihre wissenschaftliche Unterfütterung tauglich gemacht haben.

\section{Literatur}

1 Stange R: Erklärt «Verschränkung» die Komplementärmedizin? Forsch Komplementmed 2006;13: $49-51$.
Milgrom LR: Patient-practitioner-remedy (PPR) entanglement, part 8: 'Laser-Like' action of the homeopathic therapeutic encounter as predicted by a gyroscopic metaphor for the vital force. Forsch Komplementärmed Klass Naturheilkd 2005;12: 206-213.
3 Resch KL: «Die Andere Medizin»: Gut gemeint schlecht gemacht. Forsch Komplementmed 2006;13: 6-8.

4 Gigerenzer G: Bauchentscheidungen - die Intelligenz des Unbewussten und die Macht der Intuition. München, Bertelsmann, 2007. 